AROUEOLOGÍA Y SOCIEDAD

№ 26, 2013: 289-300

ISSN: $0254-8062$

RECIBIDO: 30 / MAR. / 2013

ACEPTADO: 15 / ABR. / 2013

\title{
PAISAJE RITUALY MARCADORES ASTRONÓMICOS EN EL SITIO UÑA TAMBO, NEVADOS DE CACHI, SALTA, ARGENTINA
}

\author{
«Está saliendo el sol, que es sin duda mi Dios» \\ Cristian Álvarez
}

\author{
CRISTIAN JACOB \\ Facultad de Filosofía y Letras, UniversidAd de Buenos Aires, ARgentina \\ zamapurro@yahoo.com.ar \\ IVÁN LEIBOWICZ \\ InSTITUTO MULTIDISCIPLINARIO DE HISTORIA Y CIENCIAS HUMANAS, CONICET, ARGENTINA \\ pinocarriaga@hotmail.com \\ FÉLIX ACUTO \\ Instituto Multidisciplinario de Historia y CienCIas Humanas, CONICET, ARgentina \\ acuto@gmail.com \\ RICARDO MOYANO \\ Posgrado Arqueología Escuela Nacional de Antropología e Historia, MÉxico \\ mundosubterraneo@hotmail.com
}

\section{RESUMEN}

En este trabajo daremos cuenta de las nuevas investigaciones realizadas en los Nevados de Cachi, Salta, Argentina focalizándonos en el sitio que hemos denominado Uña Tambo, y cuál fue su integración dentro de la red de sitios inkaicos de la región. En esta ocasión haremos hincapié en algunos aspectos arqueoastronómicos del asentamiento, sin dejar de lado la importancia visual y simbólica que tuvo la ubicación de esta clase de sitios así como también la relevancia de los mismos dentro de los rituales inkaicos relacionados con el agua, la agricultura y con el culto a los Apus.

Palabras ClaVE: Inka, Apus, paisaje, ritual, arqueoastronomía.

\section{Abstract}

In this paper we will realize the new research in the Nevados de Cachi, Salta, Argentina by focusing on the site that we called Uña Tambo, and what was its integration within the network of Inka sites in the region. We'll concentrate on some aspects of the settlement archaeoastronomical, without neglecting the visual and symbolic importance of the location in that kind of sites as well as their relevance within Inka rituals related to water, agriculture and the cult of Apus.

KeYwords: Inka, Apus, landscape, ritual, archaeoastronomy. 


\section{UÑA TAMBO}

El sitio que hemos decidido llamar Uña Tambo (2459'25,4"S, 66²19'17,5”W) se ubica en la vertiente oriental de los nevados de Cachi, a $4705 \mathrm{msnm}$, en la provincia de Salta en el noroeste argentino (Fig. 1). Se encuentra en la quebrada de La Hoyada, en el camino que asciende al cerro Meléndez (donde existe una plataforma ceremonial Inka) y a unos 700 metros de distancia, en línea recta, del sitio El Apunao (Jacob y Leibowicz 2011; Jacob et al. 2011), separados ambos por el llamativo promontorio conocido como La Uña (Fig. 2).

Entendemos que este sitio es aquel oportunamente identificado por Pio Pablo Díaz en los registros del Museo Arqueológico de Cachi como SSalCac 84. Asimismo Vitry (2008) rescata el comentario

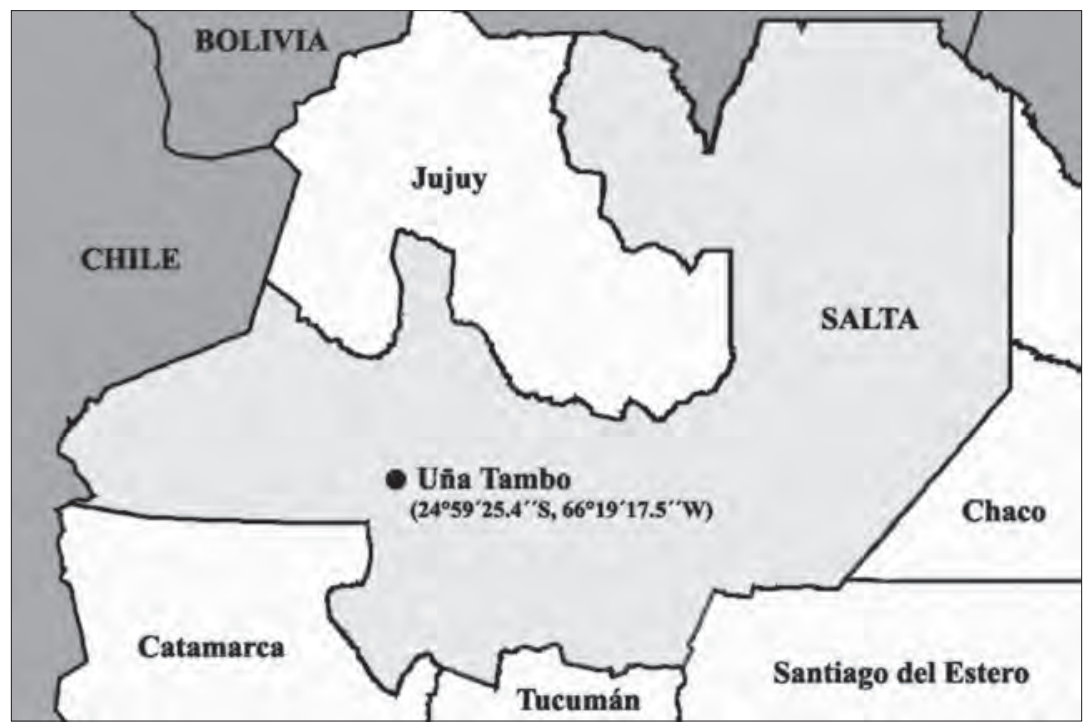

Figura 1. Ubicación geográfica del sitio Uña Tambo.

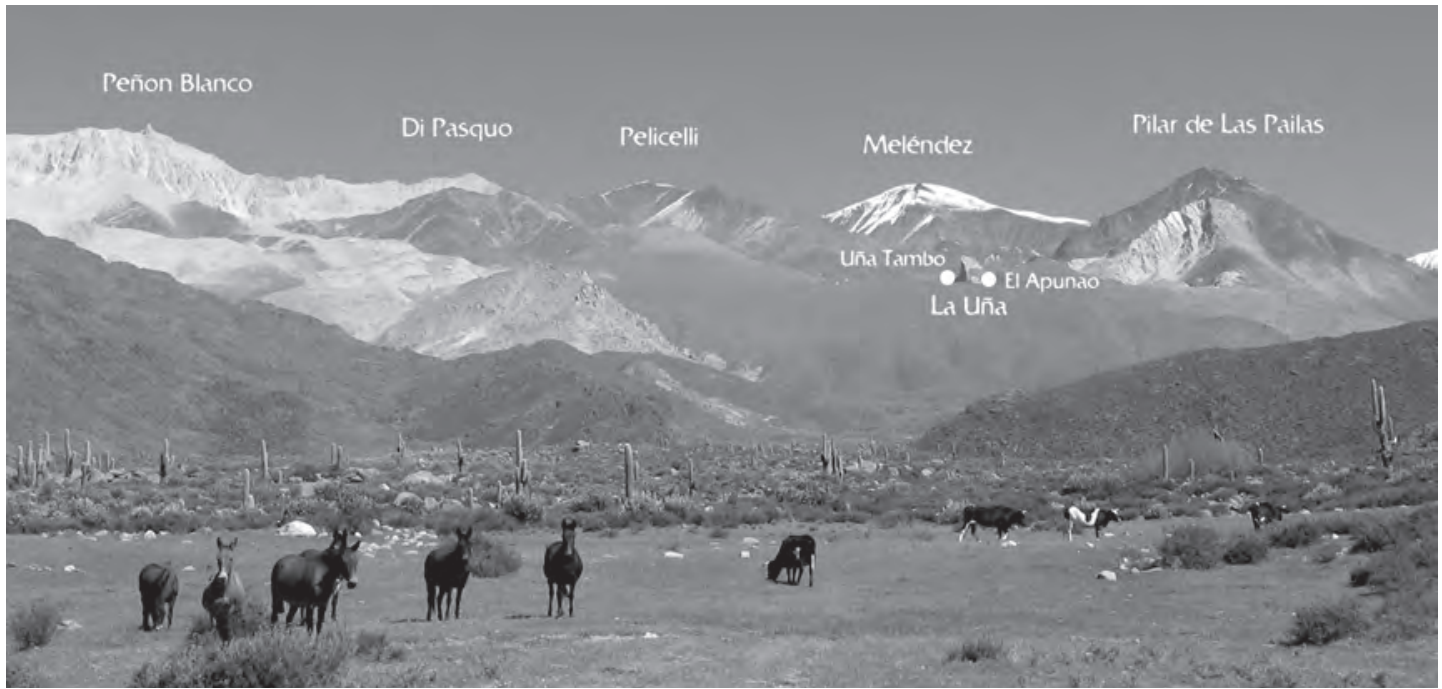

Figura 2. Vista del cordón montañoso Nevado de Cachi, con sus principales cumbres, la Uña y los sitios Uña Tambo y El Apunao. 
de una expedición del Club Andino de Bariloche, efectuada en la década de 1940 «depositamos estupefactos las mochilas, al hallarnos en medio de una aldea formada por numerosas casas de piedra, derrumbadas, con muros de hasta un metro y medio de alto, y desprovistas de techo. Este hallazgo nos sorprende bastante, pues estamos casi a 4.900 metros de altura» (Boucher 1949 en Vitry 2008). Constanza Ceruti (2009), también menciona este asentamiento al documentar su ascensión a la cima del cerro Meléndez por la quebrada de la Hoyada, destacando la presencia de cerámica bicolor y tricolor en superficie.

Con estas referencias, el asentamiento fue claramente identificado por medio de Google Earth, en el intento de relevar por este medio posibles sitios relacionados con El Apunao y el cerro Meléndez (Fig. 3). En octubre de 2011 se realizó el primer croquis del lugar, con brújula y cinta métrica, distinguiendo además de los sectores identificados por Google Earth, un tramo de camino Inka que sube por la quebrada de La Hoyada e ingresa al asentamiento por el Recinto 28.

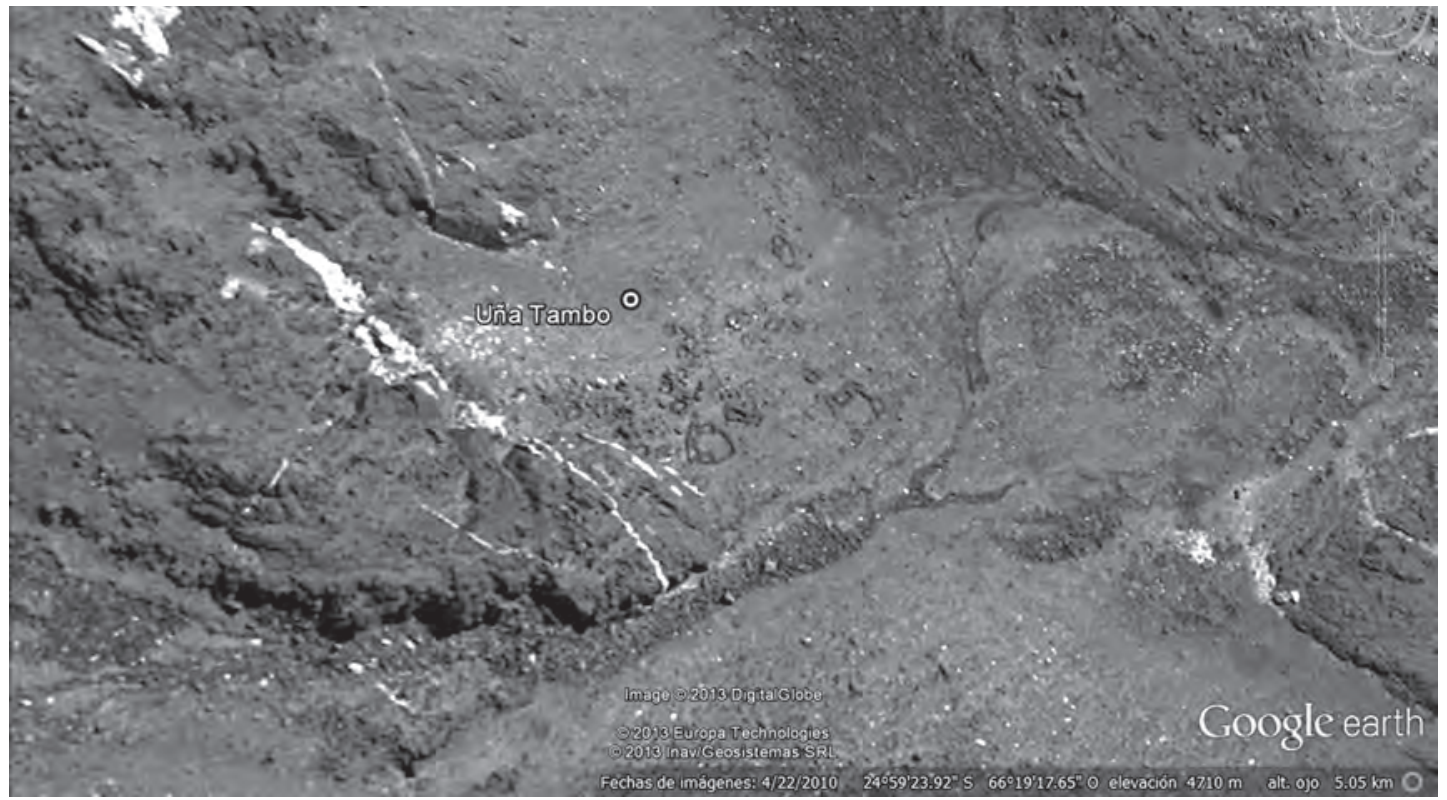

Figura 3. Imagen satelital obtenida con el programa Google Earth donde se observan las estructuras del Uña Tambo.

El sitio, que cuenta con 43 recintos (Fig. 4), se encuentra dividido en dos sectores, ubicados en diferentes cotas altitudinales. En ambos se destaca la existencia de una kancha o un rectángulo perimetral compuesto -RPC- (sensu Raffino 1981), que cuenta con un sector central despejado, tipo plaza, y recintos rectangulares, semicirculares e irregulares bordeando la misma (Fig. 5). El estilo constructivo se compone de muros simples y dobles, sin argamasa. Algunos de los recintos rectangulares, tanto en los RPC como fuera de ellos, fueron edificados con piedras que presentan algunas de sus caras planas, ángulos rectos y vanos que permitirían catalogarlos como inkaicos (sensu Raffino 1981).

En superficie se identificaron fragmentos de cerámica del estilo Santamariano pertenecientes al período de Desarrollos Regionales (1000-1450 d.C.) e Inka Provincial, entre los que se hallan fragmentos de pucos, bordes, asas y partes del cuerpo de distintos tipos de vasijas (Fig. 6). Además se registró desechos de talla tales como lascas de riolita y cuarcita.

Durante estas labores se realizó el significativo hallazgo, en el sector más alto del sitio y dentro de un pequeño recinto semicircular (Recinto 2), de una piedra vertical tipo gnomon en su pared norte (Fig. 7). 


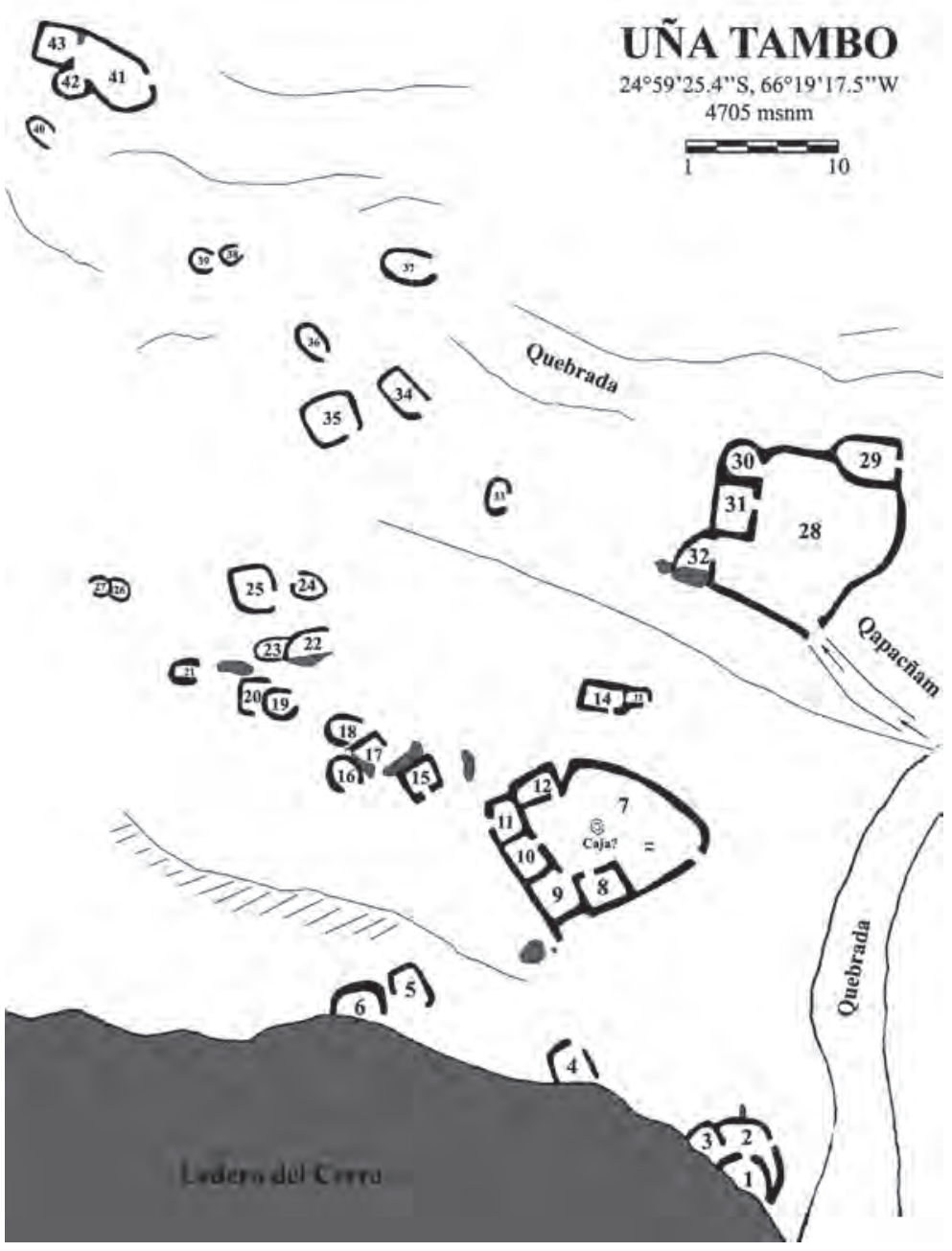

Figura 4. Plano de Uña Tambo.

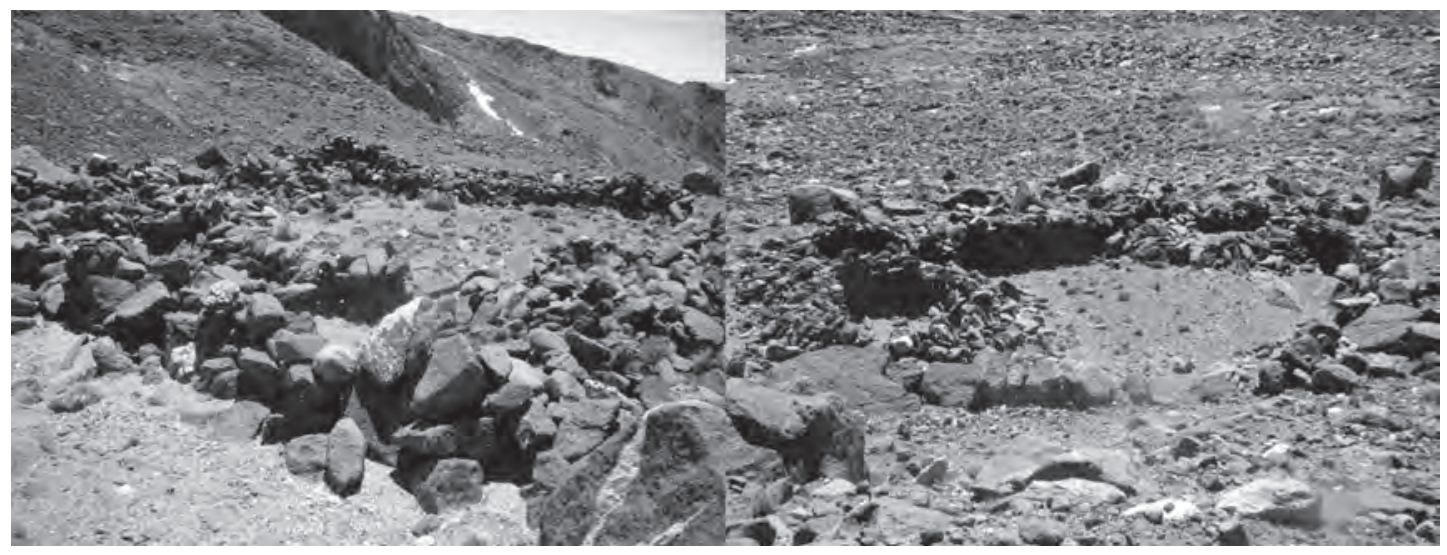

Figura 5. Recintos tipo RPC de Uña Tambo. 


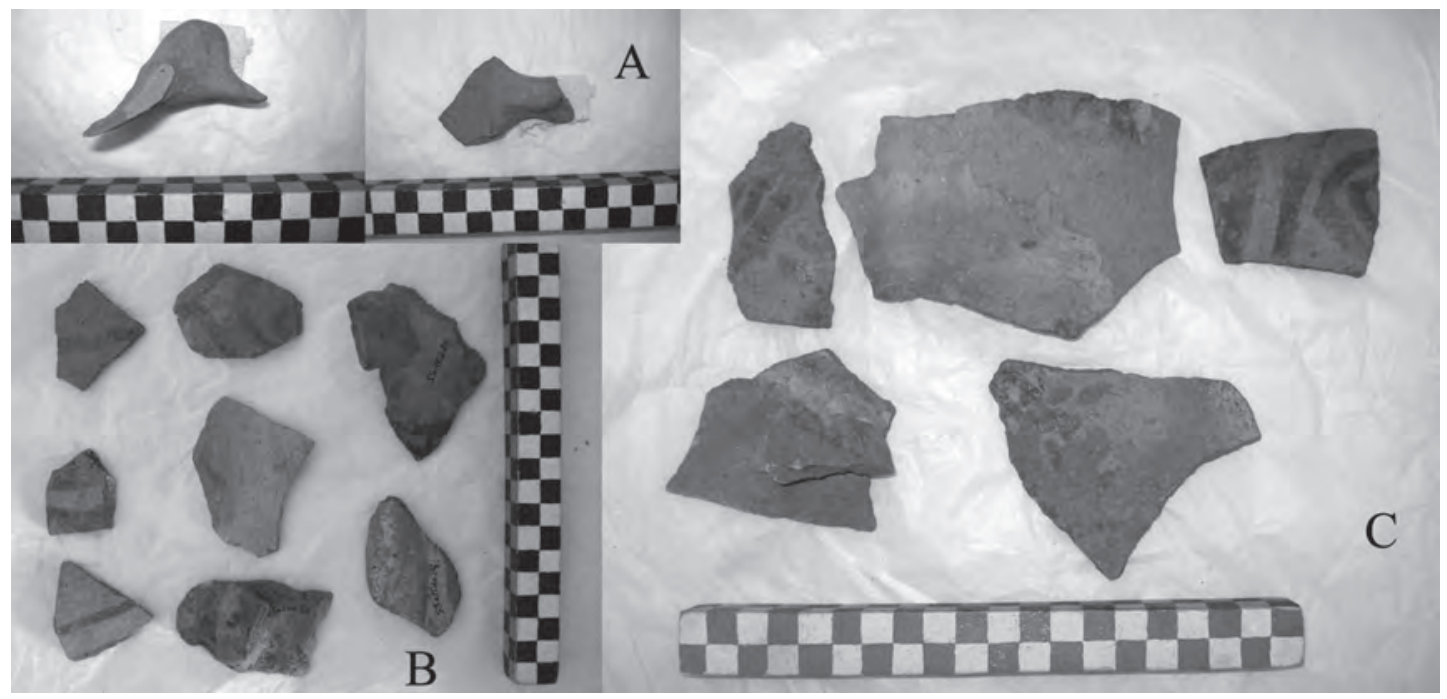

Figura 6. Cerámica hallada en superficie en Uña Tambo. A: Apéndice ornitomorfo de puco inkaico. B: Fragmentos de vasijas Santamarianas. C: Fragmentos de urna Santamariana.

\section{Cielo y TIERRA, Un PAisAJE COMPLETO}

En trabajos anteriores hemos focalizado nuestra atención en la constitución ideológica y política de diferentes especialidades y paisajes pretéritos (Acuto 1999, 2004, 2012; Jacob y Leibowicz 2011; Leibowicz 2007, 2012). Entendiendo que estos lugares donde se llevaron a cabo determinadas prácticas sociales o donde se plasmaron ciertas relaciones sociales fueron parte fundamental de las mismas, constituyéndose ambas categorías en una relación dialéctica (Soja 1989). Si bien nuestro énfasis fue colocado en investigaciones que podríamos llamar terrestres, consideramos que el cielo (el paisaje astronómico) no escapó a estas categorizaciones. Y que en la medida de lo posible debemos intentar observar y analizar este paisaje (terrestre y celeste) en su totalidad.

Por lo tanto, creemos que las observaciones astronómicas en el pasado tenían, además de su dimensión cognitiva y simbólica, una dimensión social. Las maneras de pensar y de clasificar los fenómenos astronómicos se generan, producen y representan en contextos sociales bien definidos, es decir, con una base social concreta que las ha elaborado, en muchos casos, en un campo de relaciones de poder, dominación y conflicto.

Encararemos entonces este trabajo entendiendo a la arqueastronomía como el estudio de creencias y prácticas sobre el cielo en el pasado, especialmente en la prehistoria, y los usos que la gente hizo de ese conocimiento de de los cielos (Ruggles 2005).

Figura 7. Gnomon pétreo ubicado en el Recinto 2 de Uña Tambo.

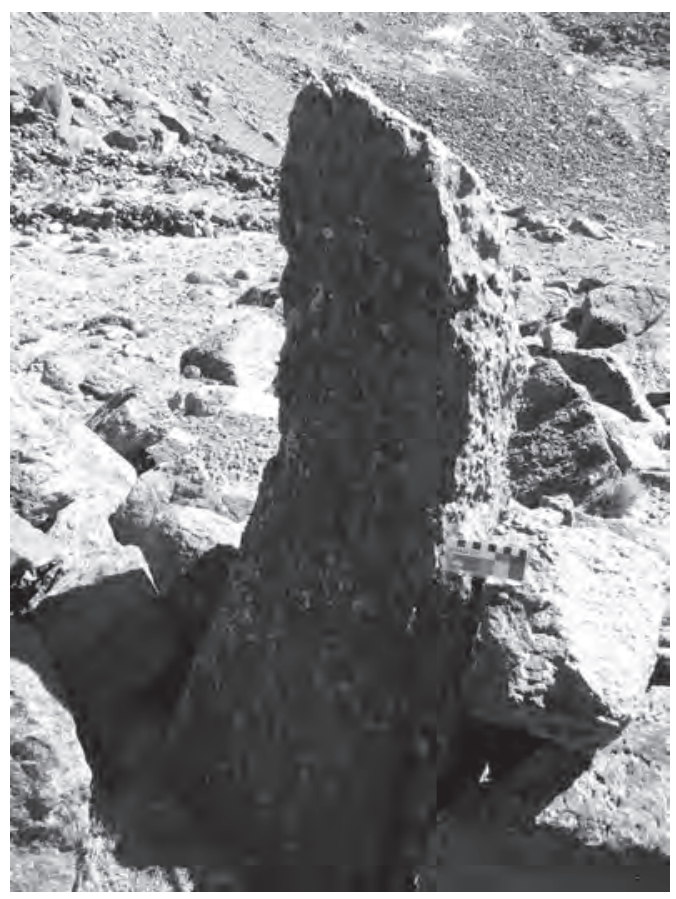


El ojo y el cerebro humano son los instrumentos astronómicos por excelencia, utilizados en conjunto con elementos culturales y naturales como la arquitectura (muros, ventanas y hornacinas), el gnomon, pozos de agua, agujeros en la tierra, cavernas, paneles de arte rupestre, torres, apachetas y elementos de la topografía, utilizados y/o transformados culturalmente como marcadores calendáricos de cenit (gnomon) y horizonte ${ }^{1}$.

Asimismo, entender al cielo como una categoría social, implica entender al mismo no como un ente abstracto, sino como el resultado de una construcción cultural que participa activamente de la vida social, en cuanto a la proyección de valores, normas y categorías socioculturales. En este contexto, los cuerpos celestes y algunos fenómenos meteorológicos, al igual que las montañas, se convierten en agentes o actores sociales, que mediante la actuación marcan y negocian su status, rango, clase, edad y género, como parte de de un campo/espacio social (Iwaniszewski 2009: 34-35). El cielo era una parte integral y prominente del cosmos percibido, sus incesantes ciclos y la familiaridad de su observación fueron parte del tejido de la vida (Ruggles 2005).

En tiempos inkaicos la observación del Sol, la Luna, las estrellas y determinadas constelaciones era parte del manejo y control de distintos tipos de calendario. Se ha postulado incluso, que las observaciones astronómicas hechas dentro y en los alrededores del Cusco fueron el núcleo de los rituales públicos más importantes del Imperio (Bauer y Dearborn 1995: 1). Para ello, se supone la existencia de especialistas que llevaban la cuenta de los días llamados camayoc o «[...] funcionarios[...] que entendían exclusivamente de realizar esas observaciones. En las provincias fueron levantados observatorios semejantes [pilares] que señalaban el tiempo de la siembra según lo que correspondía al lugar[...]» (Jesuita Anónimo 1964 Tomo II: 345, en Williams 1992: 102). Guamán Poma de Ayala 1992 [1560] los define como el «astrologo inca» y/o el «secretario» (Fig. 8). Ambos, utilizan un quipu o sistema mnemotécnico de cuerdas de lana o algodón y nudos, de uno o varios colores, para llevar la contabilidad y el control de la producción estatal. Según Zuidema (1989), éste tipo de procedimiento junto con el sistema de ceques habría servido para coordinar el año solar y los meses lunares siderales dentro del calendario inkaico.

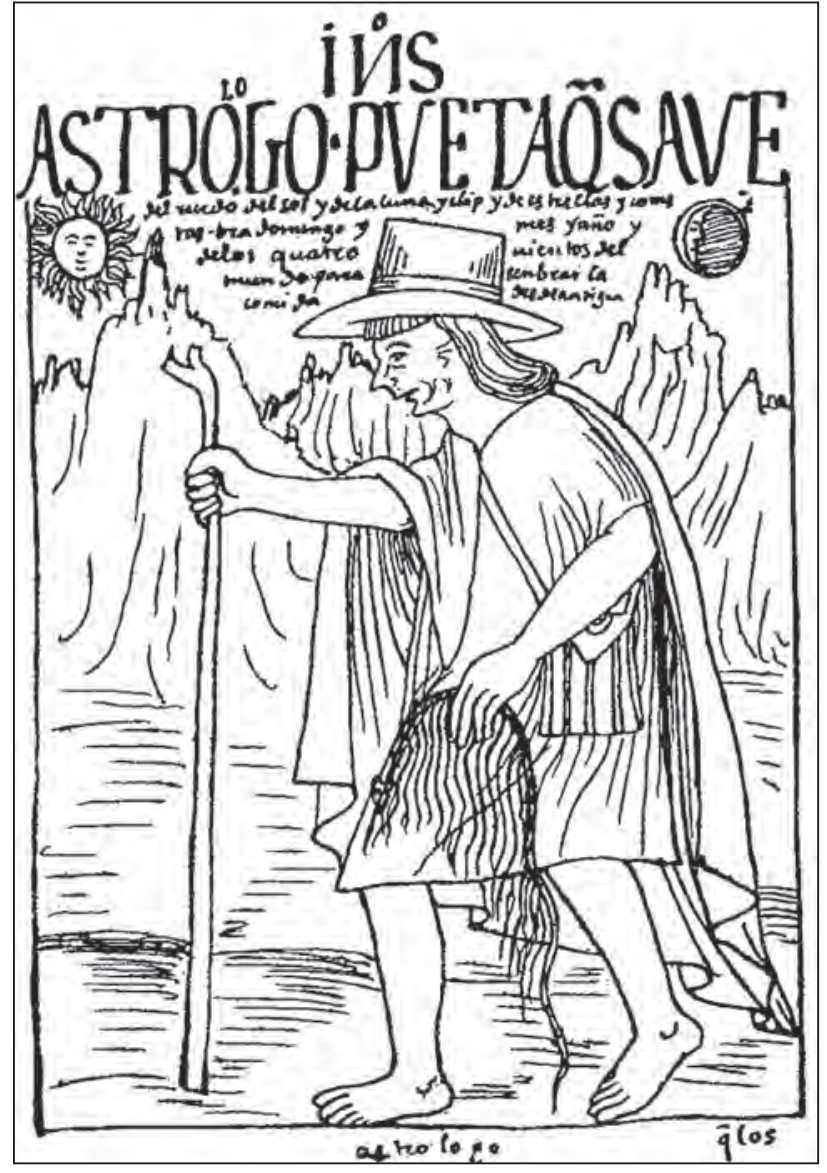

Figura 8. Astrologo Inka según Guamán Poma de Ayala.

1 Marcador: construcción(es) humanas o rasgos naturales en el paisaje utilizados (desde un punto de observación) para establecer la posición del Sol en el horizonte asociado con un evento cultural (Hardman y Hardman 1992:154). 


\section{MEDiciones ARQUEOASTRONÓMICAS}

En septiembre de 2012 se ascendió nuevamente al sitio, con el fin de observar allí el equinoccio de primavera. Como hipótesis preliminar, se intentó constatar la funcionalidad astronómica del gnomon identificado en el Recinto 2, en la parte más alta del sitio. Además se midió el acimut del cerro La Uña, el cual es el elemento más llamativo de la topografía del lugar y se encuentra ubicado al noreste del punto de observación. A la par, también se llevó a cabo la observación de la Luna en primer cuarto gracias a la fotografía digital, entre los días 21 y 23 de septiembre, consiguiendo excelente tomas gracias a las buenas condiciones de luz y visibilidad.

El teodolito se instaló a 1,70 m del gnomon, una roca vertical de cuatro lados y punta en prisma, con dimensiones de 110 x 40 × $25 \mathrm{~cm}$, inclinada levemente al norte, en dirección al cerro La Uña. La piedra se corresponde con las rocas del lugar y no cuenta con evidencias claras de canteado o trabajo. Los resultados de la medición y el cálculo de los horizontes son los siguientes:

Tabla 1. Marcadores de horizonte de Uña Tambo

\begin{tabular}{|c|c|c|c|c|c|c|c|}
\hline Punto & Nombre & Vertical (v) & $\begin{array}{c}\text { Horizontal } \\
(\mathrm{h})\end{array}$ & $\begin{array}{c}\text { Declinación } \\
(\delta)\end{array}$ & Fecha & $\begin{array}{c}\text { Observa- } \\
\text { ciones }\end{array}$ & 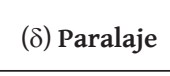 \\
\hline 1 & C. La Uña & $16^{\circ} 06^{\prime} 03,07^{\prime \prime}$ & 2732'12”" & $40^{\circ} 55^{\prime} 14,02^{\prime \prime}$ & - & - & - \\
\hline 2 & - & $18^{\circ} 18^{\prime} 17,56^{\prime \prime}$ & $330^{\circ} 50^{\prime} 12^{\prime \prime}$ & $38^{\circ} 13^{\prime} 28,1^{\prime \prime}$ & - & - & - \\
\hline 3 & $\begin{array}{c}\text { Pilar de } \\
\text { las Pailas }\end{array}$ & $21^{\circ} 03^{\prime} 31,59^{\prime \prime}$ & $339^{\circ} 38^{\prime} 12^{\prime \prime}$ & $39^{\circ} 52^{\prime} 47,25^{\prime \prime}$ & - & - & - \\
\hline 4 & - & $17^{\circ} 21^{\prime} 11,91^{\prime \prime}$ & $354^{\circ} 11^{\prime} 12^{\prime \prime}$ & $47^{\circ} 16^{\prime} 45,01^{\prime \prime}$ & - & - & - \\
\hline 5 & - & $13^{\circ} 45^{\prime} 43,11^{\prime \prime}$ & $23^{\circ} 31^{\prime} 12^{\prime \prime}$ & $44^{\circ} 58^{\prime} 6,62^{\prime \prime}$ & - & - & - \\
\hline 6 & - & $02^{\circ} 12^{\prime} 06,79^{\prime \prime}$ & $43^{\circ} 43^{\prime} 12^{\prime \prime}$ & $39^{\circ} 40^{\prime} 6,94^{\prime \prime}$ & - & - & - \\
\hline 7 & - & $(-) 01^{\circ} 05^{\prime} 36,42^{\prime \prime}$ & $71^{\circ} 47^{\prime} 12^{\prime \prime}$ & $17^{\circ} 50^{\prime} 7,11^{\prime \prime}$ & 01ago/11may & - & \\
\hline 8 & - & $(-) 01^{\circ} 01^{\prime} 14,28^{\prime \prime}$ & $92^{\circ} 51^{\prime} 12^{\prime \prime}$ & $(-) 02^{\circ} 09^{\prime} 14,7^{\prime \prime}$ & $15 \mathrm{mar} / 28 \mathrm{sep}$ & P-SSEQ & - \\
\hline 9 & - & $(-) 01^{\circ} 22^{\prime} 48,07^{\prime \prime}$ & 9803'12" & $(-) 06^{\circ} 42^{\prime} 19,99^{\prime \prime}$ & $03 \mathrm{mar} / 10 \mathrm{oct}$ & & - \\
\hline 10 & & $(-) 01^{\circ} 43^{\prime} 12,2^{\prime \prime}$ & $111^{\circ} 32^{\prime} 12^{\prime \prime}$ & $(-) 18^{\circ} 39^{\prime} 24,11^{\prime \prime}$ & $27 \mathrm{ene} / 16 \mathrm{nov}$ & SLMS & $(-) 19^{\circ} 05^{\prime} 16,59^{\prime \prime}$ \\
\hline 11 & $\begin{array}{l}\text { C. El } \\
\text { Negro }\end{array}$ & $(-) 00^{\circ} 03^{\prime} 56,28^{\prime \prime}$ & $101^{\circ} 31^{\prime} 12^{\prime \prime}$ & $(-) 10^{\circ} 24^{\prime} 1,86^{\prime \prime}$ & $22 \mathrm{feb} / 20 \mathrm{oct}$ & - & - \\
\hline
\end{tabular}

P-SSEQ: pre-salida Sol equinoccio

SLMS: Salida Luna mínima sur

- El cerro La Uña de ubica al este del norte con un acimut de 27³2'12", no coincidiendo con ningún marcador de horizonte para el Sol o la Luna.

- Al oriente se pueden identificar marcadores de horizonte para los días cercanos al equinoccio (15 de marzo y 28 de septiembre) en un cerro de nombre indeterminado (N.8) y altura negativa $\left(-01^{\circ} 01^{\prime}\right)$, con un acimut de $92^{\circ} 51^{\prime} 12^{\prime \prime}$ (P-SSEQ).

- El punto N.10 marca la posición del nodo menor con un acimut de 111³2'12" (27 de enero y 16 de noviembre, para el Sol). Este punto se acerca a la posición de la salida de la Luna en parada menor al sur con una declinación calculada (paralaje) de $-19^{\circ} 05^{\prime} 16,59^{\prime \prime}$ (SLMS).

- Por su parte el cerro El Negro, acimut 101 ${ }^{\circ} 31^{\prime} 12^{\prime \prime}$, marca las fechas del 22 de febrero y 20 de octubre.

- Al poniente no se observan marcadores de importancia, debido a las condiciones naturales de horizonte (cercanía de la pared de rocas), sólo existiendo visible el cerro Pilar de las Pailas con un acimut de $339^{\circ} 38^{\prime} 12^{\prime \prime}$.

- Desde el punto de vista solar, se constató en campo la orientación equinoccial del gnomon (Fig. 9) así como la posibilidad que esta roca vertical de cuatro lados y punta en prisma esté representándola imagen del cerro La Uña (Fig. 10).

Astronómicamente se constató la posibilidad de marcar al equinoccio en el horizonte, gracias al uso del gnomon como punto de referencia con respecto al Sol al oriente 


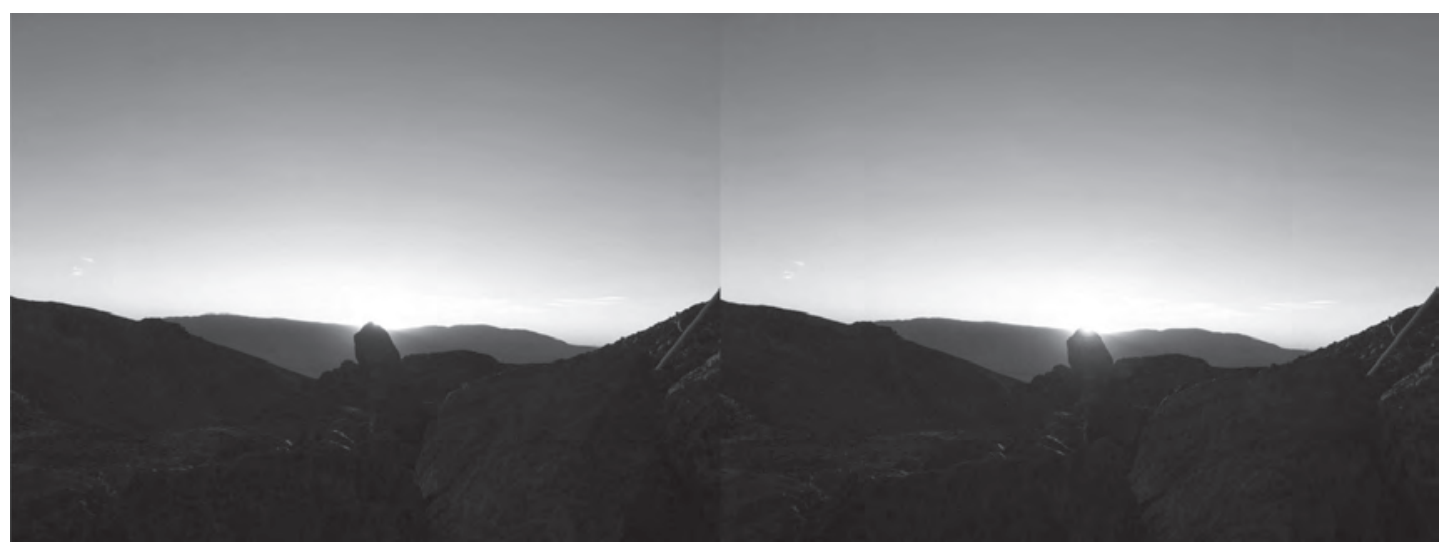

Figura 9. Gnomon de Uña Tambo en la salida del sol durante el equinoccio de primavera.

Por consecuencia, este gnomon - posiblemente- marcó los días cercanos al equinoccio y con ello, eventualmente la posición de la Luna en los meses cercanos a marzo y septiembre (fenómeno del crossover). Con lo cual podemos afirmar que dicho gnomon, cumplía una función que podría equipararse a la de una Intihuatana o lugar donde se amarra el sol.

\section{IMPORTANCIA VISUAL DE LA UÑA}

Consideramos que el sitio no solo cuenta con estas propiedades a nivel astronómico, sino que su ubicación en el paisaje, la decisión de construirlo allí, tuvo una significativa importancia.

Se ha documentado ampliamente la importancia que las montañas, particularmente aquellas con sus cumbres cubiertas de nieve tienen y han tenido en la cosmología andina (Bastien 1978; Martínez 1983; Reinhard 1985). Estos rasgos topográficos han sido identificados como entidades tutelares, Apus y pacarinas o lugar de origen de las ancestros fundadores de las comunidades.

El cerro Meléndez, debió ser, en tiempos prehispánicos, una importante Wak'a a nivel regional, el Apu que dominaba el actual valle

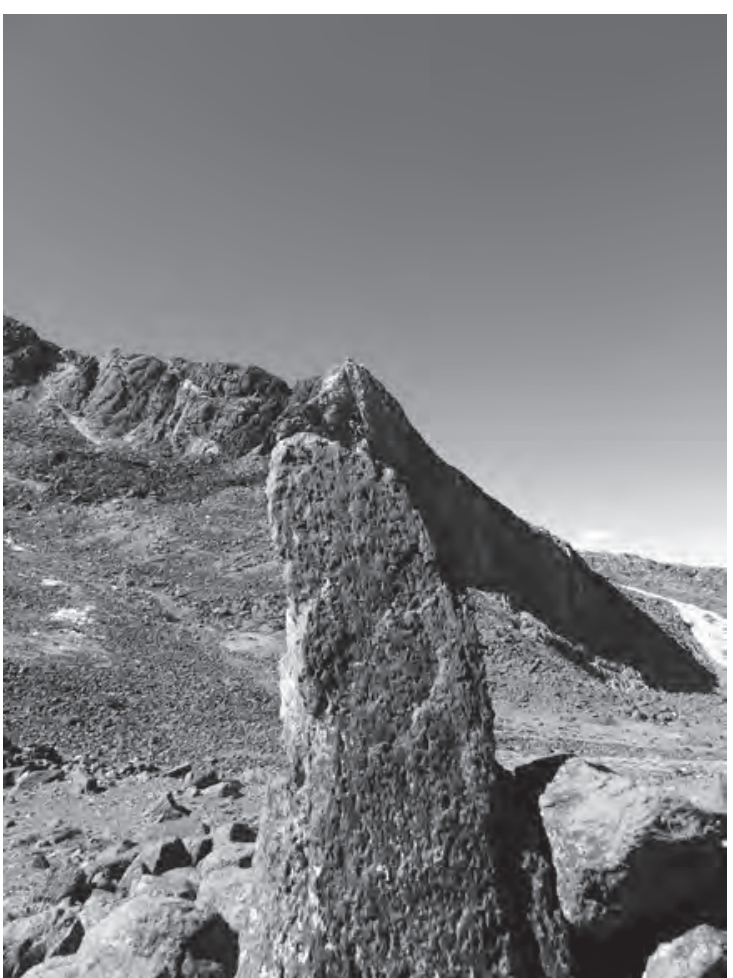

Figura 10. Gnomon de Uña Tambo y la Uña. Calchaquí Norte, la antigua provincia inkaica de Chicoana. En relación a esto, cabe destacar que el ushnu de Cortaderas Bajo, el recinto AD 10 de Cortaderas Derecha y el ushnu de Guitian así como la Casa Morada de la Paya están orientados hacia el Meléndez, lo cual nos llevaría a pensar que en la construcción de estos sitios se vieron involucrados conceptos de orden cósmico y ritual.

Uña Tambo y El Apunao, se encuentran separados, por tan solo 700 metros por el promontorio rocoso conocido localmente como la Uña. Esta notoria formación geológica se destaca no solo por su forma sino también por su color. A la distancia parece ubicarse bajo la cima nevada del Cerro Meléndez, emulando de alguna manera al Apu (ver Figura 2). Esto la hace visible desde varios de 
los sitios ubicados en el Valle Calchaquí (Cortaderas, Buena Vista, Valdez, Ambrosia Torres, Quipón, Tero, Fuerte Alto, Mariscal, Las Pailas, La Paya, Guitián, Loma del Oratorio, Borgata), e incluso desde la lejana recta del Tin Tin. Un fenómeno similar se puede observar en el cerro Aconcagua, donde en la Pirámide, un llamativo contrafuerte ubicado a $5300 \mathrm{msnm}$ en el filo suroccidental del mismo se hallaron restos humanos y materiales de una capacocha (Bárcena 1989; Gentile 1996; Schobinger 1999). Schobinger (1999: 11) analiza los motivos que llevaron a los inkas a montar allí este adoratorio y remarca que «desde la base en la Quebrada de Horcones (sector Confluencia-Playa Ancha) no se ve la cumbre del Aconcagua, tapada por la «Pirámide» que impresiona así como un cerro propio.» A partir de esto se pregunta si «¿Percibieron los súbditos incaicos un simbolismo especial en esa forma geométrica?» (Schobinger 1999: 11)

\section{DisCUSIÓN Y CONCLUSIONES}

Vemos al Uña Tambo, su construcción, su ocupación como parte de un proceso más amplio que es la conquista inkaica del Valle Calchaquí Norte. Al mismo tiempo que se construyen los grandes centros imperiales en el valle, como Potrero de Payogasta, Cortaderas, La Paya/Guitián, se produce la apropiación simbólica y efectiva de los Nevados (Acuto et al. 2010; Jacob y Leibowicz 2011). La importancia de estos, dentro de las estrategias inkas de conquista se refleja en la cantidad de asentamientos allí construidos y en los rituales y ceremonias allí efectuados.

Como ya mencionamos, existe en la cima del Cerro Meléndez una plataforma de 12,2 x 6,3 m, con los ejes mayores orientados en sentido este-oeste, donde pudo desarrollarse alguna ceremonia (capacocha?) (Vitry 2008). En El Apunao hemos documentado la existencia de un complejo ushnu conformado por una plataforma de 9 x $7 \mathrm{~m}$ y una estructura tipo baño con su piso enlajado y canaleta de drenaje, siendo este un lugar propicio para ceremonias libatorias (Jacob y Leibowicz 2011). A esto se suma la presencia, sobre el curso de agua que divide al sitio en dos, de un petroglifo con claras referencias calendáricas (Jacob et al. 2011). A su vez, la sensación visual desde donde se halla emplazado el sitio es similar a la de un escenario, evidenciando que la elección de la ubicación del mismo no quedó librada al azar, ya que desde este se domina visualmente toda la quebrada de Las Pailas y los valles inferiores circundantes (Jacob y Leibowicz 2011). Hemos interpretado a este sitio como posiblemente relacionado con el ritual agrícola, ya que allí nacen las aguas que luego formaran el río Las Pailas. Tal vez allí se realizaron festividades mas privadas, propias de aquellos enviados o autoridades imperiales.

Por otra parte, la existencia de un marcador equinoccial en Uña Tambo nos remite a la importancia de esta fecha en distintas festividades inkaicas que han sido registradas por diversos cronistas. Por ejemplo Garcilaso de la Vega destaca que los inkas observaban los equinoccios con gran solemnidad, mientras que para el equinoccio de marzo cosechaban el maíz en los campos de Cusco con gran regocijo y celebraciones, durante el equinoccio de septiembre se festejaba la Situa (Garcilaso de la Vega 1976 [1609]). Es interesante tener en cuenta que actualmente en el Valle Calchaquí las fechas de siembra y cosecha son las mismas.

Cabe aclarar que no creemos que grandes ceremonias de este tipo hayan tomado lugar en el Recinto 2 de Uña Tambo (donde se encuentra ubicado el gnomon), ya que debido a su pequeño tamaño los gnomones no pueden ser utilizados en ceremonias públicas de gran escala (Bauer y Dearborn 1995). Si en cambio podemos proponer la existencia de una suerte de observatorio astronómico ubicado en las alturas, formando parte de un complejo sistema de asentamientos dedicados a distintos tipos de rituales promovidos por el Tawantinsuyu. No obstante, la presencia de recintos de gran tamaño, uno de los cuales presenta una posible caja de ofrendas (R 7), y la mixtura de arquitectura y cerámica inka y santamariana podría indicar que este sitio, paso previo y obligado a la cima del cerro Meléndez, conto con la presencia de las sometidas poblaciones locales, ya sea como constructores del asentamiento y/o como participantes de algún tipo de ceremonial, ligado seguramente a las observaciones astronómicas que allí se realizaron. Además, la elección de construir dos sitios de características rituales como Uña Tambo y El 
Apunao claramente vinculados con una formación geológica como La Uña, tuvo la marcada intencionalidad de relacionarlos con un elemento destacado del paisaje. Siendo este un marcador a la distancia de las actividades que los conquistadores Inkas desarrollaban en los nevados.

Nos encontramos entonces con tres sitios que presentándose como expresión de un mismo fenómeno, fueron posiblemente utilizados de manera diversa, y por distintas personas o grupos sociales, siendo cada uno de ellos el responsable de diferentes tipos de ceremonias o festividades. Por todo ello, creemos que es importante continuar con las investigaciones en esta dirección, con el fin de entender el modo en que se dio esta interacción entre los inkas y las poblaciones locales.

Los nevados de Cachi, como espacio significativo del paisaje norcalchaquí, experimentaron bajo el dominio inkaico una transformación substancial. Paisajes y espacios que pudieron ser visitados o parcialmente ocupados en los Desarrollos Regionales, son domesticados por los Inkas. Las imponentes alturas que otrora fueran veneradas desde lo bajo, son colonizadas por los enviados del Tawantinsuyu, quienes crean un nuevo paisaje, una nueva relacionalidad entre los hombres y las cosas. De modo que las experiencias de las sociedades conquistadas cambian de un modo radical (Acuto 2007), conjugando en estas experiencias rituales, antiguas creencias y espacios con el novedoso escenario político regional.

Esta nueva geografía ritual (sensu Broda 2007) genera, promueve y reproduce nuevas y desiguales relaciones sociales en la región. Y la conquista, tanto simbólica como efectiva de los Nevados por parte de los Inkas jugó un papel primordial en ello.

\section{Agradecimientos}

Queremos agradecer en primer lugar a aquellas personas que de una u otra manera nos ayudaron a realizar estos trabajos de campo: Guillermo, María del Rosario y Teresa. A Marisa Kergaravat y Alejandro Ferrari por ayudarnos a armar la logística y compartir alojamiento y transporte en la segunda campaña. Y principalmente a todas las comunidades del Valle Calchaquí Norte que tan bien nos han tratado siempre. 


\section{BiBIOGRAFÍA}

ACUTO, Felix A.

1999 «Paisaje y dominación: La constitución del espacio social en el Imperio Inka». En: A. Zarankin y F.A. Acuto (eds.) Sed Non Satiata. Teoría Social en la Arqueología Latinoamericana Contemporánea: 33-75. Buenos Aires: Ediciones Del Tridente.

2004 Landscapes of Ideology and Inequality: Experiencing Inka Domination. Ph.D. Dissertation, State University of New York - Binghamton.

2007 «Fragmentación vs. Integración comunal: Repensando el Período Tardío del Noroeste Argentino». Estudios Atacameños, Arqueología y Antropología Surandinas 34: 71-96.

2012 «Landscapes of inequality, spectacle and control: Inka social order in provincial contexts». Revista Chilena de Antropología 25: 9-64.

ACUTO, Félix A., Andrés TRONCOSO, Alejandro FERRARI, Daniel PAVlOVIC, Cristian JACOB, Ezequiel GILARDENGHI, Rodrigo SÁNCHEZ, Claudia AMUEDO y Marina SMITH

2010 «Espacialidad Incaica en los Andes del Sur: La colonización simbólica del paisaje y la ritualidad Inca en Chile Central y el Valle Calchaquí Norte». En: J. R. Bárcena y H. Chiavazza (eds.) Arqueología Argentina en el Bicentenario de la Revolución de Mayo, 1810-2010, Tomo 3: 1297-1302. Mendoza.

BÁRCENA, Joaquín Roberto

1989 «Pigmentos en el ritual funerario de la momia del cerro Aconcagua». Xama 2: 61-116.

BASTIEN, Julian

1978 Mountain of the condor. Metaphor and ritual in an Andean ayllu. Illinois: Waveland Press, Inc. Prospect Heights.

BAUER, Brian y David DEARBORN

1995 Astronomy and Empire in the Ancient Andes. The Cultural Origins of Inca Sky Watching. Austin: University of Texas Press.

BRODA, Johanna

2007 «Astronomía y paisaje ritual: el calendario de horizonte de Cuicuilco-Zacatepetl». En: J. Broda, S. Iwaniszeski y A. Montero (eds.) La Montaña en el paisaje ritual: 173-199. México: ENAH, CONACULTA/ INAH, IIH-UNAM.

CERUTI, Constanza

2009 «Relevamiento arqueológico en el Nevado de Cachi: Cumbre Meléndez y Quebrada de la Hoyada (Provincia de Salta)». Actas del VI Congreso Argentino de Americanistas, Tomo 2: 17-32. Buenos Aires: Sociedad Argentina de Americanistas.

DÍAZ, Pío Pablo

1983 «Sitios arqueológicos del valle calchaquí». Estudios de Arqueología № 3 y 4. Museo Arqueológico de Cachi.

GARCILASO DE LA VEGA, Inca

1976 [1609] Comentarios Reales de los Incas. Aurelio Miró Quesada (prólogo, edición y cronología). Biblioteca Ayacucho, Colección Clásica, 2 Vols.: Tomo I (№ 5) y Tomo II (№ 6). Caracas. Venezuela.

GENTILE, Margarita

1996 «Dimensión sociopolítica y religiosa de la capacocha del cerro Aconcagua». Boletín del Instituto de Estudios Andinos 25 (1): 43-90.

GUAMAN POMA DE AYALA, Felipe

1992 [1560] Nueva Crónica y Buen Gobierno. México: Siglo XXI.

HARDMAN, Clark Jr. y HARDMAN, Marjorie H.

1992 «Linear solar observatory theory: the development of concepts of time and calendar». North American Archaeologist 13(2), 149-172.

IWANISZEWSKI, Stanislaw

2009 «Por una astronomía cultural renovada». Complutum 20 (2): 23-37. 
JACOB, Cristian e Ivan LEIBOWICZ

2011 «Montañas sagradas en los confines imperiales. Nevado de Cachi, Salta-Argentina». Revista Haucayapata. Investigaciones arqueológicas del Tahuantinsuyo 2: 71-90.

JACOB, Cristian, Ricardo MOYANO, Félix ACUTO e Ivan LEIBOWICZ

2011 «Quilca del cielo: Valle Calchaquí, Salta, Argentina». Boletín APAR 3 (10): 348-350.

LEIBOWICZ, Ivan

2007 «Espacios de poder en La Huerta, Quebrada de Humahuaca». Estudios Atacameños, Arqueología y Antropología Surandinas 34: 51-70.

2012 «Ideología y Espacio: Conquista Inka en la Quebrada de Humahuaca, Jujuy, Argentina». Revista Chilena de Antropología 25: 65-91.

MARTÍNEZ, Gabriel

1983 «Los dioses de los cerros en los Andes». Journal de la Societé des Americanistes 85: 85-115.

RAFFINO, Rodolfo

1981 Los Inkas del Kollasuyu. La Plata: Ramos Americana Editora.

REINHARD, Johan

1985 «Sacred mountains: an ethnoarchaeological study of high Andean ruins». Mountain Research and Development 5(4): 299-317.

RUGGLES, Clive

2005 Ancient Astronomy: An Encyclopedia of Cosmologies and Myth. ABC-CLIO

SCHOBINGER, Juan

1999 «Los santuarios de altura incaicos y el Aconcagua: aspectos generales interpretativos». Relaciones 24: 7-27.

SOJA, Edward

1989 Postmodern geographies. The reassertion of space in critical social theory. Verso, Londres y Nueva York.

VITRY, Cristian

2008 El Nevado de Cachi. Río Cuarto: Palloni Ediciones.

WILLIAMS, Carlos

1992 «Sukankas y Ceques, la Medición del Tiempo en el Tahuantinsuyo». Revista del Museo de la Nación, I (1): 101- 113.

ZUIDEMA, R.Tom

1989 «El ushnu». En M. Burga (comp.), Reyes y Guerreros: Ensayos de la Cultura Andina: 402-454. Lima: Ediciones Fomciencias. 


\title{
CONSTRUYENDO UN PAISAJE INKA: LA CONVERSIÓN DE LOS CENTROS CEREMONIALESY LA CONSTITUCIÓN DE LA AUTORIDAD DURANTE LA FORMACIÓN DEL ESTADO INKAIKO (CUSCO, PERÚ)
}

\author{
Steve Kosiba, Ph.D. \\ Departamento de ANTRopología, Universidad de Alabama \\ sbkosiba@as.va.edu \\ Vicentina GaLiano Blanco \\ MINISTERIO DE CULTURA, CUSCO \\ vikig7@hotmail.com
}

\section{RESUMEN}

En el artículo se presentarán datos arqueológicos recientes de prospecciones y excavaciones realizadas en el área de Ollantaytambo, para examinar cómo los grupos sociales de la región del Cusco fueron incorporados al Estado inka. En particular, se analizará como los Inkas afirmaron su autoridad transformando el paisaje de la región, remodelando la arquitectura de lugares ceremoniales preexistentes y regulando las actividades rituales tradicionales, lo que conlleva un cuestionamiento de las teorías predominantes sobre la formación del Estado inka.

Palabras Clave: Paisaje, Sistema de Información Geográfica, Inka, Periodo Intermedio Tardío, Cusco.

\section{Abstract}

En el artículo se presentarán datos arqueológicos recientes de prospecciones y excavaciones realizadas en el área de Ollantaytambo, para examinar cómo los grupos sociales de la región del Cusco fueron incorporados al Estado inca. En particular, se analizará como los Incas afirmaron su autoridad transformando el paisaje de la región, remodelando la arquitectura de lugares ceremoniales preexistentes y regulando las actividades rituales tradicionales, lo que conlleva un cuestionamiento de las teorías predominantes sobre la formación del Estado inca.

KeYwords: Paisaje, Sistema de Información Geográfica, Inka, Periodo Intermedio Tardío, Cusco. 